\title{
Determinación de la constante de Planck mediante el método experimental con la utilización de diodos emisores de luz leds
}

Determination of the Planck constant through the experimental method with the use of diodes led transmitters

Recibido: marzo 15 de 2017 | Revisado: abril 10 de 2017 | Aceptado: mayo 15 de 2017

Gian Carlos Scarpati ${ }^{\mathrm{I}}$

Resumen

El siguiente artículo describe la manera de calcular la constante de Planck a través de la pendiente de la recta obtenida de la medida de la energía cinética máxima como función de la frecuencia. Para este experimento se emplea diodos emisores de luz que emiten en diferentes frecuencias de la zona visible del espectro. Finalmente, se determina la constante de Planck con una buena aproximación (por debajo del 3\%).

Palabras Clave: efecto fotoeléctrico, constante de Planck, diodos emisores de luz, relatividad, física cuántica

\begin{abstract}
The following article describes how to calculate the Planck constant as the slope of the line of the graph of maximum kinetic energy as function of frequency via light-emitting diodes emitting at different frequencies in the visible region of the spectrum.
\end{abstract}

Key words: photoelectric effect, Planck constant, light emitting diodes, relativity, quantum physics

1 Universidad de San Martín de Porres, Perú

gscaspatig@usmp.pe 


\section{Introducción}

La mecánica cuántica es una de las más excitantes ramas de la física. Tuvo sus inicios a principios del siglo XX, cuando dos de las teorías que intentaban explicar lo que nos rodea, la ley de gravitación universal y la teoría electromagnética clásica se volvían insuficientes para explicar ciertos fenómenos que sucedían a nivel microscópico.

Fue Max Planck quien entonces enunció la hipótesis de que la radiación electromagnética es absorbida y emitida por la materia en forma de «cuantos» de luz o fotones de energía mediante una constante estadística, que se denominó la Constante de Planck.

La idea de Planck habría quedado muchos años solo como hipótesis si Albert Einstein no la hubiera retomado, proponiendo que la luz, en ciertas circunstancias, se comporta como partículas de energía independientes (los cuantos de luz o fotones).

Einstein completó en 1905 las correspondientes leyes de movimiento, las que se conocen como teoría especial de la relatividad. Demostró así que el electromagnetismo era una teoría, esencialmente, no mecánica. Culminaba, de este modo, lo que se ha dado en llamar física clásica, es decir, la física no-cuántica.

Las aplicaciones tecnológicas derivadas de la aplicación de la mecánica cuán- tica suponen, en mayor o menor medida, el 30 por ciento del PIB de los Estados Unidos.

\section{Consideraciones teóricas}

La constante de Planck es la relación entre la cantidad de energía y la frecuencia asociadas a un cuanto o a una partícula. En la ecuación $\mathrm{E}=\mathrm{hV}$, E es la energía del fotón emitido, $\nabla$ (letra griega nu) es la frecuencia de la luz del diodo emisor de luz y $h$ es la constante de Planck. E es a su vez, igual al producto de (e) $(\mathrm{Vg})$, siendo $\mathrm{Vg}$ el voltaje de frenado y e $=$ $1,602 \times 10^{-19} \mathrm{C}$, es la carga elemental del electrón. (Beiser,1994)

\section{Determinación del voltaje de frenado}

El Voltaje de frenado $(\mathrm{Vg})$ se obtiene implementando el circuito (Figura 1). Donde la fuente de luz consiste en un arreglo de tres diodos emisores de luz (leds). Este arreglo se replica para tres colores: rojo, amarillo y verde. Como superficie fotoeléctrica se utiliza una celda solar obtenida de una lámpara solar de jardín doméstica. El procedimiento consiste en obtener el voltaje de frenado correspondiente a cada arreglo de led's de distinto color. Este voltaje se mide directamente del voltímetro, girando el potenciómetro hasta hacer que la corriente a medir en el amperímetro sea cero. Se recomienda poner el miliamperímetro en la escala más baja para obtener valores más precisos en la obtención del voltaje de frenado. 


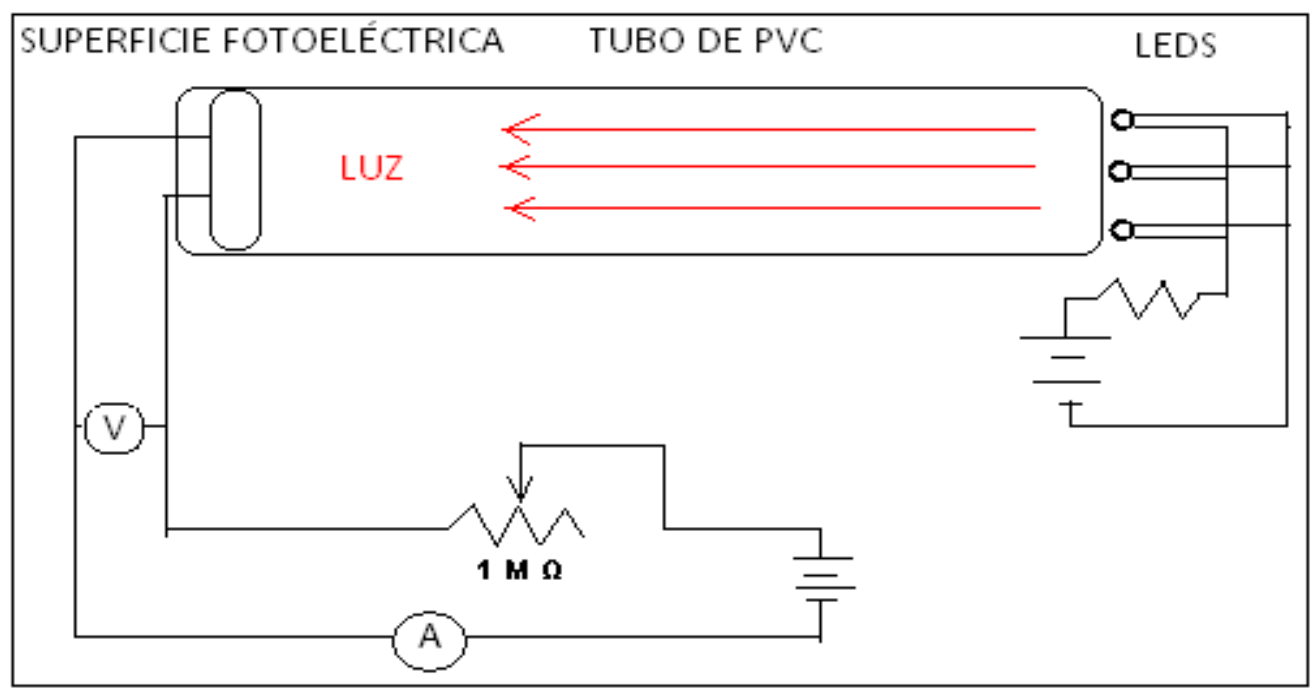

Figura 1.Circuito electrónico utilizado para la determinar la constante de Planck.

\section{Determinación de la constante de Planck}

Se elaboró el cuadro mostrado en la Figura 2, en la cual el valor de la energía potencial se contrasta con la frecuencia de la luz que emite cada LED. El valor de la longitud de onda $V$ correspondiente a cada color de LED se observa en la Figura 3.
El valor de la velocidad de la luz, $\mathrm{C}$ se divide entre valor de longitud de onda $\mathrm{V}$ para hallar la frecuencia de la luz emitida por cada LED. Haciendo uso de la estimación lineal, se determina las estadísticas de una línea utilizando el método de «mínimos de cuadrados», para calcular la línea recta que mejor se ajuste a los datos obtenemos un valor de $\mathrm{h}=6,821 \times 10^{-34}$.

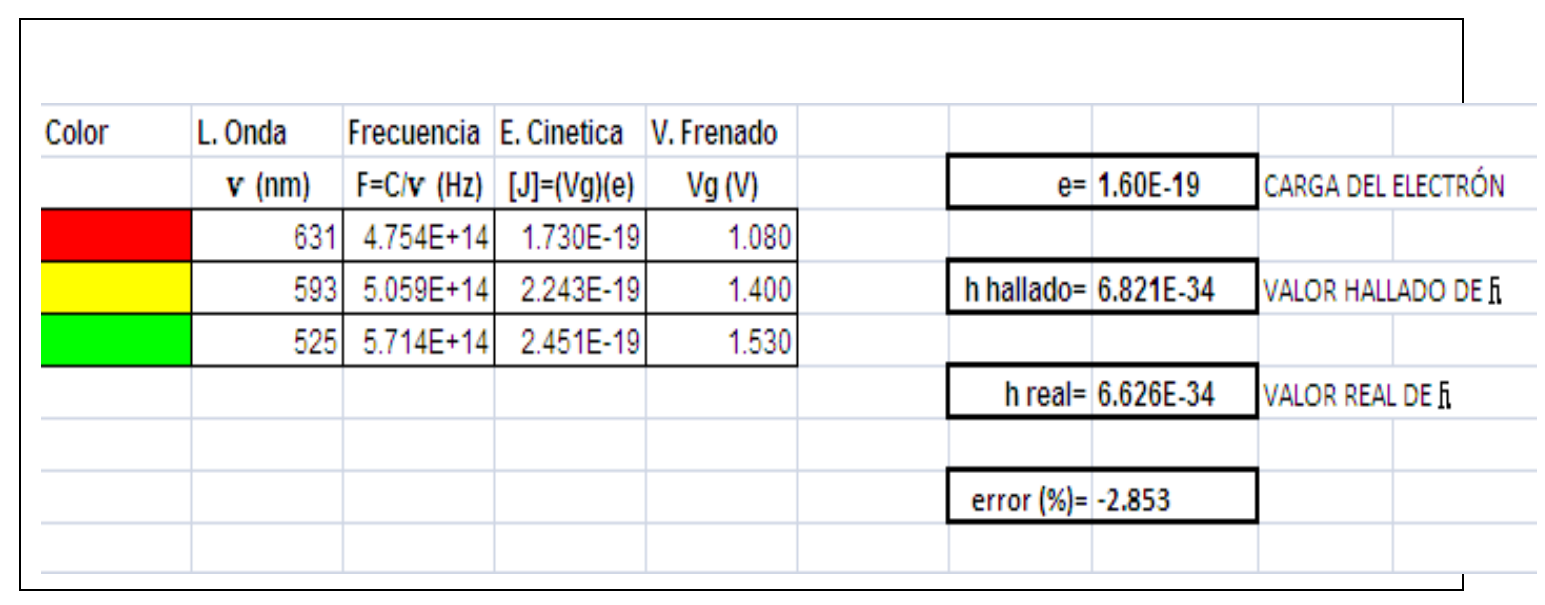

Figura 2. Cálculo de la constante de Planck

\section{Conclusiones}

El valor obtenido se aproxima al valor real de la constante, atribuyéndose la diferencia a errores experimentales en la medición.
Queda demostrado de que es posible en la práctica, y con materiales sencillos, obtener un valor considerablemente cercano al esperado. 


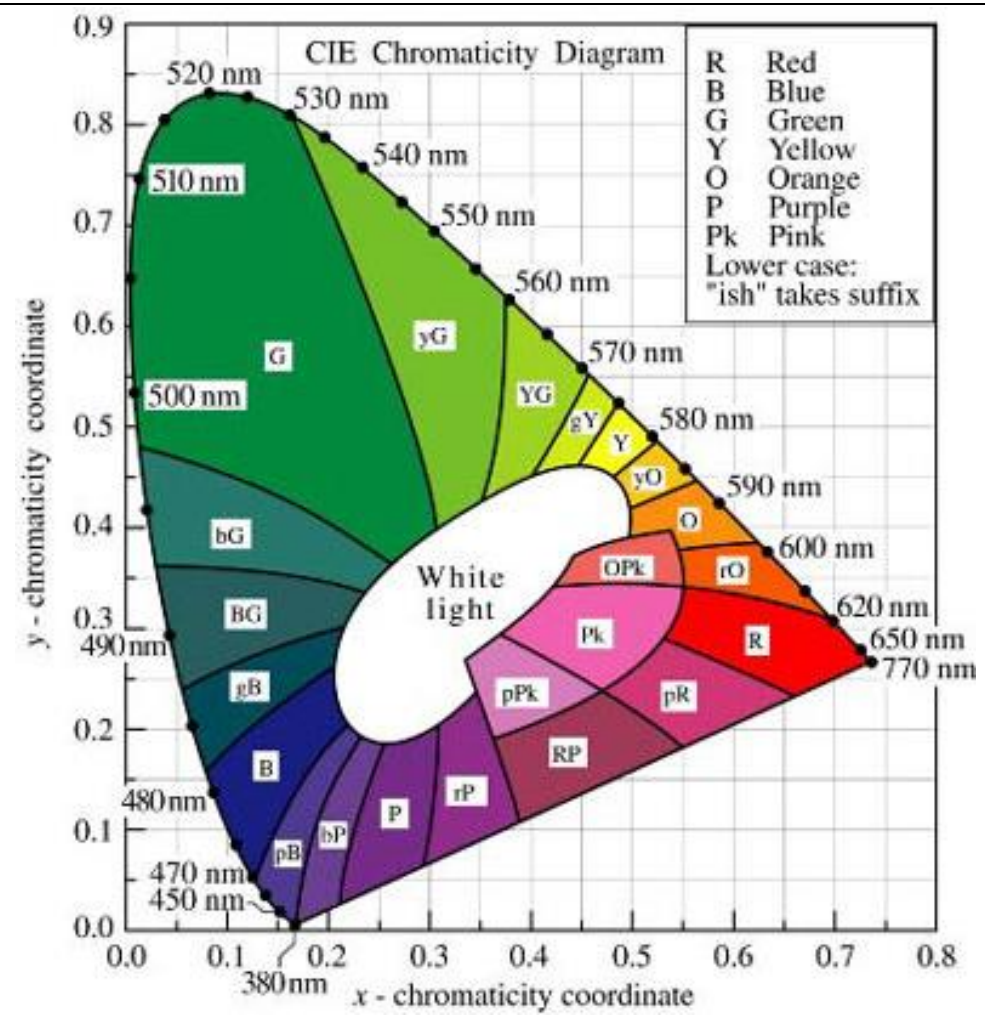

Figura 3. Disgrama de cromaticidad

\section{Referencias}

Beiser, A. (1994). Conceptos de Física moderna. México. Ed. Mc GrawHill. 